\title{
TWENTY MONTHS OF EVOLUTION FOLLOWING SYMPATHECTOMY ON PATIENTS WITH PALMAR HYPERHIDROSIS: SYMPATHECTOMY AT THE T3 LEVEL IS BETTER THAN AT THE T2 LEVEL
}

\author{
Guilherme Yazbek, ${ }^{I}$ Nelson Wolosker, ${ }^{\mathrm{I}}$ Paulo Kauffman, ${ }^{\mathrm{I}}$ José Ribas Milanez de \\ Campos, Pedro Puech-Leão, ${ }^{\mathrm{I}}$ Fábio Biscegli Jatene ${ }^{\mathrm{II}}$
}

doi: 10.1590/S1807-59322009000800006

\begin{abstract}
Yazbek G, Wolosker N, Kauffman P, de Campos JR, Puech-Leão P, Jatene FB. Twenty months of evolution following sympathectomy on patients with palmar hyperhidrosis: sympathectomy at the $\mathrm{t} 3$ level is better than at the $\mathrm{t} 2$ level. Clinics. 2009;64(8):743-9.
\end{abstract}

OBJECTIVE: To compare two surgical techniques (denervation levels) for sympathectomy using video-assisted thoracoscopy to treat palmar hyperhidrosis in the long-term.

METHODS: From May 2003 to June 2006, 60 patients with palmar hyperhidrosis were prospectively randomized for video-assisted thoracoscopic sympathectomy at the T2 or T3 ganglion level. They were followed for a mean of 20 months and were evaluated regarding their degree of improvement of palmar hyperhidrosis, incidence and severity of compensatory hyperhidrosis and its evolution over time, and quality of life.

RESULTS: Fifty-nine cases presented resolution of the palmar hyperhidrosis. One case of therapeutic failure occurred in the T3 group. Most of the patients presented an improvement in palmar hyperhidrosis, without any difference between the groups. Twenty months later, all patients in both groups presented some degree of compensatory hyperhidrosis but with less severity in the T3 group $(p=0.007)$. Compensatory hyperhidrosis developed in most patients during the first month after the operation, with incidence and severity that remained stable over time. An improvement in quality of life was seen starting from the first postoperative evaluation but without any difference between the groups. This improvement was maintained until the end of the follow-up.

CONCLUSION: Both techniques were effective for treating palmar hyperhidrosis. The most frequent complication was compensatory hyperhidrosis, which presented stable incidence and severity over the study period. Sympathectomy at the T3 level presented compensatory hyperhidrosis with less severity. Nevertheless, the improvement in quality of life was similar between the groups.

KEYWORDS: Palmar hyperhidrosis; Compensatory hyperhidrosis; Video-assisted thoracoscopy; Symphatectomy; Quality of life .

\section{INTRODUCTION}

Palmar hyperhidrosis is a somatic disorder characterized by excessive sudoresis that is limited to the hands. It is caused by hyperfunctioning of the sympathetic nervous system and is frequently related to a triggering emotional

${ }^{\text {I }}$ Department of Vascular Surgery, Hospital das Clínicas, Faculdade de Medicinada Universidade de São Paulo - São Paulo/SP, Brazil

II Thoracic Surgery Service, Division of Thoracic Surgery, Hospital das Clínicas da Faculdade de Medicina da Universidade de São Paulo - São Paulo/SP, Brazil

Email: guilhermeyazbek@yahoo.com

Tel: 55112063.5577

Received for publication on April 16, 2009

Accepted for publication on May 04, 2009 situation. ${ }^{1,2}$ This hyperfunction goes beyond what is needed for thermoregulation. ${ }^{3,4}$ It may cause serious problems of psychosocial nature ${ }^{2,3}$ and also professional difficulties, as these individuals may develop conditions of social phobia. ${ }^{5}$

The only effective and definitive treatment for essential hyperhidrosis is sympathectomy. The excellent functional results with this treatment mean that it is currently considered the best therapeutic approach. ${ }^{1,2,3,4,6}$

As the operation has become technically simpler, the number of patients undergoing sympathectomy has been increasing. Consequently, compensatory hyperhidrosis is seen more frequently. ${ }^{7,8}$ Of all the complications associated with sympathectomy, this compensatory hyperhidrosis is the most common and, when severe, may cause dissatisfaction 
regarding the operation. The relationship between the severity of compensatory hyperhidrosis and the denervation level is still unclear and has not been investigated by prospective randomized studies..$^{9,10,11}$ The aim of the present prospective randomized study was to compare the results from sympathectomy performed at the T2 and T3 denervation levels with 20 months of follow-up, by analyzing the resolution of palmar hyperhidrosis, incidence and severity of compensatory hyperhidrosis and its evolution over time, and patients' quality of life.

\section{MATERIAL AND METHODS}

This prospective, randomized and controlled study was conducted in accordance with the norms of the Ethics Committee for Analysis of Research Projects involving Human Experimentation, and all subjects gave their informed consent. From May 2003 to June 2006, sixty patients with palmar hyperhidrosis were included, underwent sympathectomy and were followed up. The surgical treatment was selected randomly. Patients with prior thoracic surgery, cardiac diseases, pulmonary infections, neoplasia or pleural or lung diseases that could increase surgical risk, or with a body mass index (BMI) greater than 25 were not included in this study.

Twenty-four of the patients had undergone previous attempts at clinical treatment. Most of these (18 cases) had used local dermatological therapies (creams and lotions). Lack of success had made them seek other therapeutic possibilities for resolving their hyperhidrosis.

Half of the 60 patients were randomly assigned to undergo thoracic sympathectomy by means of videothoracoscopy at the denervation level of the T2 ganglion and the other half at the T3 level. None of the patients were aware of the sympathectomy level used.

The patients' demographic characteristics are presented in Table 1.

Table 1 - Clinical characteristics of patients with palmar hyperhidrosis

\begin{tabular}{lccc}
\hline & T2 & T3 & $p$-value \\
\hline Mean age & $23.4 \pm 5.3$ & $23.2 \pm 6.5$ & $0.5833(\mathrm{NS})$ \\
$\begin{array}{l}\text { Number of } \\
\text { women/men }\end{array}$ & $24 / 6$ & $17 / 13$ & 0.052 (NS) \\
\hline NS - not significant & & & \\
\hline
\end{tabular}

There was no statistically significant difference in relation to the variables of sex and age.

The patients underwent the operations under general anesthesia in a semi-seated position $\left(45^{\circ}\right)$. Two mini- incisions of approximately $5.5 \mathrm{~mm}$ each were made: the first into the fourth or fifth intercostal space on the anterior axillary line (in the submammary sulcus for the women), through which a video camera was introduced, and the second into the second intercostal space on the mid-axillary line, through which an electric or harmonic bistoury was introduced. The level of ganglion resection was randomly assigned; half of the patients were resected at the T2 level and the other half at the T3 level. After identification of the sympathetic chain in the patients drawn for the $\mathrm{T} 2$ group, it was sectioned (sympathicotomy) on the body of the second and third ribs, followed by thermoablation of the segment isolated between the ribs. The patients drawn for the T3 group underwent sectioning of the sympathetic chain (sympathicotomy) on the body of the third and fourth ribs, followed by thermoablation of the segment isolated between the ribs. The same procedure was performed on the contralateral chain.

The patients underwent clinical assessment on five occasions: before the operation for their inclusion in the study, one week, one month, six months and at least twelve months after the operation. The last assessment was occurred between 12 and 34 months after the operation, with a mean of 20 months. All the assessments were made in person by the principal investigator. All the questionnaires were answered without any medical interference.

During the postoperative follow-up, the following parameters were evaluated:

1. Presence or absence of palmar anhydrosis via the patient's report and confirmation from physical examination;

2. Presence or absence of compensatory hyperhidrosis, with evaluation of location and severity when present via the patient's report and confirmation from physical examination. The severity of the compensatory hyperhidrosis reported spontaneously by the patients was graded as severe or non-severe. Severe compensatory hyperhidrosis was considered to consist of visible and embarrassing compensatory sudoresis that necessitated more than one change of clothing during the day.

3. The patients' degree of satisfaction was measured by means of the quality-of-life protocol described by Amir et al. ${ }^{12}$. In this protocol, situations of daily life in which hyperhidrosis might interfere were put forward. Quality of life was initially classified into five different satisfaction levels, and obtained as the summed total score from the protocol (range from 20 to 100). When the total was greater than 84 , the quality of life was considered very poor; from 69 to 84 , poor; from 52 to 68 , good; from 36 to 51 , very good; and from 20 to 35 , excellent. The post-operative evaluation (one month, six months and 
20 months) was classified into five different satisfaction levels, and obtained as the summed total score from the questionnaire. When the total was greater than 84 , the quality of life was considered much worse; from 69 to 84, a little worse; from 52 to 68 , the same; from 36 to 51, a little better; and from 20 to 35 , much better. ${ }^{12,13}$

Statistical analysis:

The chi-square or Fisher exact test was used to investigate the association between the type of surgery and the possible results and complications by means of $2 \times 2$ contingency tables. The statistical tests comparing the types of surgery with the variables of interest (palmar anhydrosis, incidence and severity of compensatory hyperhidrosis and quality of life) were performed for each of the assessment times. The significance level was taken to be $5 \%$.

\section{RESULTS}

During the immediate postoperative period, all the patients reported palmar anhydrosis. One patient presented transitory right-side Horner's syndrome, probably due to heat transmission while releasing adherent tissue at the apex of the lung. A follow-up visit one month after the operation showed that this condition presented complete regression. Only one patient was not discharged from the hospital on the day following the operation (due to thoracic drainage).

There were two patients in the $\mathrm{T} 2$ group and one in the T3 group who were lost from follow-up.

We observed that all 30 patients who underwent sympathectomy at the T2 level and 29 of the 30 patients in the T3 group presented palmar anhydrosis at the return visits (one month, six months and 20 months). One patient in the $\mathrm{T} 3$ group did not present any improvement in palmar hyperhidrosis, even at the return visit one month after the operation. With a diagnostic hypothesis of technical failure, this patient underwent a new operation using thermoablation at the same level (T3) but without improvement of the palmar hyperhidrosis. Thus, we had one case of therapeutic failure in the T3 group. Currently, this patient is receiving clinical treatment with anticholinergics and has achieved a small clinical improvement in the hyperhidrosis. All 28 patients in the T2 group and 28 patients in the T3 group who were still undergoing follow-up 20 months after the operation (three cases lost from follow-up) continued to present palmar anhydrosis.

The incidence of compensatory hyperhidrosis observed during the patients' evolution is shown in Table 2.

One month after the operation, compensatory hyperhidrosis was observed in 26 patients in the T2 group $(86.66 \%)$ and in 27 in the T3 group (90\%). Six months after the operation, all patients in the $\mathrm{T} 2$ group had some degree of compensatory hyperhidrosis and only one patient in the T3 group did not present it (96.6\%). Twenty months after the operation, all patients in both groups presented some degree of compensatory hyperhidrosis.

The degree of severity of the compensatory hyperhidrosis is described in Table 3.

The patients in the $\mathrm{T} 3$ group presented a lower degree of compensatory hyperhidrosis in the assessments one month $(p<0.001)$, six months $(p=0.033)$ and 20 months after the operation $(p=0.007)$.

Compensatory hyperhidrosis occurred in different regions of the body, including the abdomen, back, gluteal region and legs. Most of the patients presented an association of compensatory hyperhidrosis locations, such that the most frequent combination was between locations on the abdomen and back. The preferential location was similar between the groups.

The situations that triggered compensatory sweating were excessive heat, physical exercise, or both. A small

Table 2 - Incidence of compensatory hyperhidrosis among patients who underwent sympathectomy

\begin{tabular}{lcccccc}
\hline Compensatory hyperhidrosis & \multicolumn{2}{c}{ 1 month } & \multicolumn{2}{c}{ 6 months } & \multicolumn{2}{c}{ 20 months } \\
\cline { 2 - 6 } & T2: T3 & $P$ & T2: T3 & $P$ & T2:T3 & $p$ \\
\hline With CH & $26: 27$ & $>0.999$ & $30: 29$ & NA & $28: 29$ & NA \\
Without CH & $4: 3$ & & $0: 1$ & & $0: 0$ & \\
\hline
\end{tabular}

NA: could not be evaluated; $\mathrm{CH}$ : compensatory hyperhidrosis

Table 3 - Degree of severity of compensatory hyperhidrosis after the operation

\begin{tabular}{lcccccc}
\hline & \multicolumn{2}{c}{ 1 month } & \multicolumn{2}{c}{ 6 months } & \multicolumn{2}{c}{ 20 months } \\
\cline { 2 - 6 } & $\mathrm{T} 2: \mathrm{T} 3$ & $p$ & $\mathrm{~T} 2: \mathrm{T} 3$ & $P$ & $\mathrm{~T} 2: \mathrm{T} 3$ & $p$ \\
\hline Non-severe & $16: 27$ & $<0.001$ & $20: 26$ & 0.033 & $15: 25$ & 0.007 \\
Severe & $10: 0$ & & $10: 3$ & & $13: 4$ & \\
\hline
\end{tabular}


number of the patients reported that manifestations relating to stress appeared.

The patients' degree of satisfaction, measured through the quality-of-life protocol, is presented in Table 4.

Table 4 - Total scores from the quality-of-life questionnaire

\begin{tabular}{lccc}
\hline Variable & T2 & T3 & $\boldsymbol{p}$ \\
\hline Initial (median) & $92(67-100)$ & $87.5(75-100)$ & 0.3052 \\
1 month (median) & $23(20-47)$ & $23(20-42)$ & 0.8423 \\
6 months (median) & $24(20-56)$ & $24(19-52)$ & 0.3905 \\
20 months (median) & $26(20-52)$ & $29(21-43)$ & 0.7609 \\
\hline
\end{tabular}

We found that initially, the quality of life was very poor in both groups (median greater than 84 ) without any statistically significant difference between them. One month after the operation, both groups considered their quality of life much better, without any significant difference between them $(p=0.8423)$. These values were maintained six months after the operation $(p=0.3905)$ and 20 months after the operation $(p=0.7609)$.

\section{DISCUSSION}

Before videothoracoscopy was developed, the surgical techniques used for performing thoracic sympathectomy had high morbidity rates. Consequently, clinical treatment was frequently used despite the unsatisfactory results. Videothoracoscopic sympathectomy has become the surgical technique of choice today because it is a minimally invasive technique that is safe and esthetically acceptable ${ }^{3,10}$. Various papers published on the use of this technique for treating hyperhidrosis have presented very encouraging success rates. ${ }^{3,13,14,15}$

Although there is no evidence for any difference in the prevalence of palmar hyperhidrosis between the sexes ${ }^{16}$, women usually seek treatment more frequently and therefore end up undergoing sympathectomy more often. $1,3,5,8,14,17,18$ This is perhaps because of greater discomfort relating to esthetics as compared to men. In our study as well, the patients were predominantly female (41 women versus 19 men).

Similar to other studies on this subject, most of our patients initially showed symptoms during childhood, ${ }^{16,19}$ and more than half had evidence of family distribution and history. ${ }^{3,16}$

Because of the early clinical presentation for individuals with hyperhidrosis, most studies have assessed very young populations, usually in their second and third decades of life. In our study, the patients were also young, with a mean age of $23.4 \pm 5.3$ years (T2) and $23.2 \pm 6.5$ years (T3). There are many advantages to carrying out a surgical procedure on young and healthy patients, but one negative factor in conducting any scientific study on such patients is that this is an age at which individuals frequently migrate. Loss from follow-up is a possibility observed in prospective studies, ${ }^{20}$ and this also occurred in our sample, with the loss of three patients (two from the T2 group and one from the T3 group).

When performing sympathectomy at the $\mathrm{T} 2$ level, the T2 ganglion is probably resected in most cases, as suggested by Ramsaroop et al., who studied 22 cadavers and demonstrated that the second ganglion was in fact located in the second intercostal space. ${ }^{21}$ In addition, Chung et al. observed from dissecting 27 cadavers that the second thoracic ganglion (T2) was most commonly (50\%) located in the middle of the second intercostal space, and in $78.8 \%$ of cases it was easily observed in the space between the lower edge of the second rib and the upper edge of the third rib. ${ }^{22}$ According to Kim, from analysis of images from 44 cases of sympathectomy using videothoracoscopy, the third ganglion was located in the third intercostal space in $59.1 \%$, at the upper edge of the fourth rib in $36.4 \%$, or on the fourth rib in $4.5 \% .^{23}$ Therefore, by sectioning the chain on the third and fourth ribs and performing thermoablation of the segment between them, we were dealing with the T3 ganglion in the great majority of cases.

For many years, it was believed that the ideal treatment for palmar hyperhidrosis would be sympathectomy on the T2 ganglion or on $\mathrm{T} 2$ and $\mathrm{T} 3$, because it was thought that the $\mathrm{T} 2$ ganglion would be the only one responsible for sympathetic innervation of the upper limbs. It would thus be the "key" ganglion for sympathetic denervation. ${ }^{18}$ The need for a combined approach towards the $\mathrm{T} 2$ and $\mathrm{T} 3$ ganglia has been based on the description of the Kuntz nerve (postganglionic fibers that would go from the T2 or T3 ganglion to the brachial plexus) ${ }^{24}$. For this reason, some authors have advocated sympathectomy at the levels of the T2 and T3 ganglia, ${ }^{25}$ while others have proposed more extensive approaches going from $\mathrm{T} 2$ to $\mathrm{T} 4 .{ }^{14} \mathrm{In}$ recent studies such as that by Lin and Telaranta, which was based on postoperative follow-up, the anatomical knowledge and denervation level used for hyperhidrosis treatment have been discussed again. ${ }^{26}$

Among palmar hyperhidrosis patients for whom the primordial factor in the treatment would be palmar anhydrosis, a lower denervation level might be associated with lack of success and high recurrence rates. ${ }^{17}$ In our sample, we only observed therapeutic failure in one case in the T3 group. Initial treatment failure is mostly related to inadequate sympathectomy, and a second operation is indicated to resolve the condition ${ }^{8}$. Despite surgical reintervention, our patient did not show any improvement in the palmar hyperhidrosis. This leads us to believe that in 
a small number of patients, a "neurological escape route" depending on T2 fibers or postganglionic T2 fibers may exist and thereby maintain palmar hyperhidrosis when such individuals undergo thoracic sympathectomy at the T3 ganglionic level. None of our patients presented recurrence of symptoms 20 months after the operation; this may have been because we sectioned the sympathetic chain in the segments above and below the selected ganglion, and then performed thermoablation on the segment thus isolated. This procedure was more complete and wide-ranging, and it impeded nerve fiber regeneration. The latter is more frequently observed in cases of sympathicotomy than in ca ses of sympathectomy. ${ }^{27}$

Horner's syndrome has become a rare complication today because the technique has developed, surgical teams have become more experienced and video equipment providing adequate viewing of the sympathetic chain is now used. ${ }^{28}$ Its occurrence is limited to cases of indirect lesion of the stellate ganglion (T1) due to heat diffusion or excessive traction of the chain. ${ }^{19,27}$ Like other authors, we believe that by performing sympathectomy aimed at ganglia further away from T1 (for example, T3), the incidence of this syndrome should diminish, as was seen in our sample of patients. The only case of Horner's syndrome was in a patient in the T3 group who presented adherent tissue that needed to be detached from one of the lungs. Heat transmission during this procedure led to the appearance of Horner's sign, but it had completely regressed by the time of the one-month follow-up.

Is our sample of patients, just as in the majority of studies in the recent literature, compensatory hyperhidrosis was the most frequent complication. This is also the complication that is most feared because it may lead to postoperative dissatisfaction among patients..$^{14,17,29,30}$ Its incidence has been variable in the studies dealing with this topic. ${ }^{3,11,14,15,17,18}$ In our study, it initially occurred more frequently among the cases in the $\mathrm{T} 2$ group, but at the end of the follow-up (20 months), all the cases that had undergone sympathectomy in both groups presented some degree of compensatory hyperhidrosis.

We believe that the high incidence of compensatory hyperhidrosis seen among our patients may be related to the tropical climate of Brazil, where temperatures are high for the greater part of the year. This consequently leads to greater sweating. Another reason for the high incidence was the more conservative definition used for classifying hyperhidrosis as compared to other authors. At present, there is no consensus for standardizing the definition of compensatory hyperhidrosis. For this reason, we used a more wide-ranging criterion: the presence of sudoresis in parts of the body where it did not exist prior to surgery, even if such sweating was observed only under specific conditions (intense heat or physical exercise).

The classification used in our study was more objective and simpler than that described by Gossot, ${ }^{17}$ who defined four different levels of severity of compensatory hyperhidrosis (absent; mild or intermittent; embarrassing, in which sudoresis is visible; and incapacitating, in which there is the need for more than one change of clothing per day). We believe that the the incapacitating level of severity might interfere with quality of life, and therefore classified our patients into two levels of severity: severe (incapacitating) and non-severe.

There seems to be a correlation between the severity of the compensatory hyperhidrosis and the extent and level of the resection in the sympathectomy. ${ }^{8,18,26,31,32,33}$ In our study, only one ganglion (T2 or T3) was resected in each of the groups; thus this was a homogenous series with respect to resection extent. Consequently, we can state that the obtained results were related only to resection level, which seems to be the most important factor for predicting the severity of the compensatory hyperhidrosis when evaluating the surgical technique. ${ }^{8,26,27}$

Katara et $\mathrm{al}^{10}$ recently published a study that compared ablation of the $\mathrm{T} 2$ ganglion with ablation of $\mathrm{T} 2$ and $\mathrm{T} 3$; there was no difference in the results with regard to efficacy (100\% success), recurrence (no cases), incidence of compensatory hyperhidrosis ( $80 \%$ of the cases), severity of the compensatory hyperhidrosis (no severe cases) and satisfaction subsequent to the procedure $(>80 \%)$.

Corroborating the data from that study, Miller et al. ${ }^{11}$ retrospectively analyzed 50 cases that underwent sympathicotomy at the T2 rib. It was found that $99 \%$ of the cases presented therapeutic success and that compensatory hyperhidrosis only occurred in six patients (12\%), of which four experienced sudoresis at rest and two after exercise.

The data obtained from our study differ from the above data, since there was high and similar incidence of compensatory hyperhidrosis in the $\mathrm{T} 2$ and $\mathrm{T} 3$ groups (100\%). Our data corroborate studies that correlate the severity of compensatory hyperhidrosis with higher resection levels; there was greater incidence of severe compensatory hyperhidrosis $(46.4 \%)$ in the T2 group as compared to $13.8 \%$ in the T3 group at the end of the follow-up ( $\mathrm{p}=0.007)$.

Several studies have reported an improvement in quality of life following sympathectomy, despite the painful postoperative period and the presence of compensatory hyperhidrosis in most cases. ${ }^{34,35}$ In both groups in our study, the preoperative quality of life was very poor, without any difference between the groups (median of 92 in the T2 group and 87.5 in T3). After the operation, we observed a notable improvement in quality of life (from 92 to 26 in the T2 
group and from 87.5 to 29 in T3). Despite the difference in severity of compensatory hyperhidrosis between the groups, there was no difference in clinical satisfaction subsequent to the procedure, as seen through the notable improvements in quality of life in both groups one month and six months after the operation (median of 23 after one month and 24 after six months, in both groups), and 20 months after the operation (median of 26 in the T2 group and 29 in T3).

The best way of dealing with compensatory hyperhidrosis would be to not cause it. ${ }^{11}$ Indications for hyperhidrosis treatment need to be made carefully and patients need to be advised regarding the results of the operation and its complications, and the fact that no perfect surgical technique is currently available. ${ }^{17}$ Adequate and detailed information for patients will lead to increased satisfaction after the operation; this is an essential part of the treatment. ${ }^{13}$

\section{CONCLUSION}

Sympathectomy at the T2 and T3 levels provided adequate long-term treatment for palmar hyperhidrosis. The most frequent complication was compensatory hyperhidrosis, which presented chronologically stable incidence and severity over the course of the study. Despite the greater severity of compensatory hyperhidrosis in the $\mathrm{T} 2$ group, the two groups presented similar improvements in quality of life, probably related to achieving palmar anhydrosis. Because of the lower severity of compensatory hyperhidrosis, we prefer the $\mathrm{T} 3$ level for treating palmar hyperhidrosis.

\section{ACKNOWLEDGEMENTS}

The authors appreciate the hard work contributed by Dr. Inês Nokubo Nishimoto.

\section{REFERENCES}

1. Kauffman P, Cinelli M, Jr, Wolosker M, Leao LE. Treatment of palmar hyperhidrosis by cervico-thoracic sympathectomy. AMB Rev Assoc Med Bras. 1978;24:29-30.

2. Montessi J, Almeida EP, Vieira JP, Abreu Mda M, Souza RL, Montessi $\mathrm{OV}$. Video-assisted thoracic sympathectomy in the treatment of primary hyperhidrosis: a retrospective study of 521 cases comparing different levels of ablation. J Bras Pneumol. 2007;33:248-54.

3. Byrne J, Walsh TN, Hederman WP. Endoscopic transthoracic electrocautery of the sympathetic chain for palmar and axillary hyperhidrosis. Br J Surg. 1990;77:1046-9.

4. Leao LE, de Oliveira R, Szulc R, Mari Jde J, Crotti PL, Goncalves JJ. Role of video-assisted thoracoscopic sympathectomy in the treatment of primary hyperhidrosis. Sao Paulo Med J. 2003;121:191-7.

5. Hehir DJ, Brady MP. Long-term results of limited thoracic sympathectomy for palmar hyperhidrosis. J Pediatr Surg. 1993;28:90911.

6. de Campos JR, Wolosker N, Takeda FR, Kauffman P, Kuzniec S, Jatene FB, et al. The body mass index and level of resection: predictive factors for compensatory sweating after sympathectomy. Clin Auton Res. 2005;15:116-20.

7. Lin TS, Fang HY. Transthoracic endoscopic sympathectomy in the treatment of palmar hyperhidrosis--with emphasis on perioperative management (1,360 case analyses). Surg Neurol. 1999;52:453-7.

8. Hsu CP, Shia SE, Hsia JY, Chuang CY, Chen CY. Experiences in thoracoscopic sympathectomy for axillary hyperhidrosis and osmidrosis: focusing on the extent of sympathectomy. Arch Surg. 2001;136:1115-7.
9. Kopelman D, Hashmonai M. The correlation between the method of sympathetic ablation for palmar hyperhidrosis and the occurrence of compensatory hyperhidrosis: a review. World J Surg. 2008;32:2343-56.

10. Katara AN, Domino JP, Cheah WK, So JB, Ning C, Lomanto D. Comparing T2 and T2-T3 ablation in thoracoscopic sympathectomy for palmar hyperhidrosis: a randomized control trial. Surg Endosc. 2007 Apr 3.;21:1768-71.

11. Miller DL, Force SD. Outpatient microthoracoscopic sympathectomy for palmar hyperhidrosis. Ann Thorac Surg. 2007;83:1850-3.

12. Amir M, Arish A, Weinstein Y, Pfeffer M, Levy Y. Impairment in Quality of Life Among Patients Seeking Surgery for Hyperhidrosis (Excessive Sweating): Preliminary Results. Isr Psychiatry Relat Sci. 2000;37:25-31.

13. de Campos JR, Kauffman P, Werebe Ede C, Andrade Filho LO, Kusniek S, Wolosker N, et al. Quality of life, before and after thoracic sympathectomy: report on 378 operated patients. Ann Thorac Surg. 2003;76:886-91.

14. Leseche G, Castier Y, Thabut G, Petit MD, Combes M, Cerceau O, et al. Endoscopic transthoracic sympathectomy for upper limb hyperhidrosis: limited sympathectomy does not reduce postoperative compensatory sweating. J Vasc Surg. 2003;37:124-8.

15. Yazbek G, Wolosker N, de Campos JR, Kauffman P, Ishy A, Puech-Leao P. Palmar hyperhidrosis--which is the best level of denervation using video-assisted thoracoscopic sympathectomy: T2 or T3 ganglion? J Vasc Surg. 2005;42:281-5.

16. Ro KM, Cantor RM, Lange KL, Ahn SS. Palmar hyperhidrosis: evidence of genetic transmission. Ibid. 2002;35:382-6. 
17. Gossot D, Toledo L, Fritsch S, Célérier M. Thoracoscopic sympathectomy for upper limb hyperhidrosis: looking for the right operation. Ann Thorac Surg. 1997;64:975-8.

18. O'Riordain DS, Maher M, Waldron DJ, O'Donovan B, Brady MP. Limiting the anatomic extent of upper thoracic sympathectomy for primary palmar hyperhidrosis. Surg Gynecol Obstet. 1993;176:151-4.

19. Kao MC, Lin JY, Chen YL, Hsieh CS, Cheng LC, Huang SJ. Minimally invasive surgery: video endoscopic thoracic sympathectomy for palmar hyperhidrosis. Ann Acad Med Singapore. 1996;25:673-8.

20. Chiou TS. Chronological changes of postsympathectomy compensatory hyperhidrosis and recurrent sweating in patients with palmar hyperhidrosis. J Neurosurg Spine. 2005;2:151-4.

21. Ramsaroop L, Singh B, Moodley J, Partab P, Satyapal KS. Anatomical basis for a successful upper limb sympathectomy in the thoracoscopic era. Clin Anat. 2004;17:294-9.

22. Chung IH, Oh CS, Koh KS, Kim HJ, Paik HC, Lee DY. Anatomic variations of the T2 nerve root (including the nerve of Kuntz) and their implications for sympathectomy. J Thorac Cardiovasc Surg. 2002;123:498-501.

23. Kim do H, Hong YJ, Hwang JJ, Kim KD, Lee DY. Topographical considerations under video-scope guidance in the T3,4 levels sympathetic surgery. Eur J Cardiothorac Surg. 2008;33:786-9.

24. Kuntz A, Alexander WF, Furcolo CF. Complete sympathetic denervation of the upper extremity. Ann Surg. 1938;107:25-31.

25. Shih CJ, Lin MT. Thermoregulatory sweating in palmar hyperhidrosis before and after upper thoracic sympathectomy. J Neurosurg. 1979;50:88-94.

26. Lin CC, Telaranta T. Lin-Telaranta classification: the importance of diferent procedures for different indications in sympathetic surgery. Ann Chir Gynaecol. 2001;90:161-6.
27. Yoon do H, Ha Y, Park YG, Chang JW. Thoracoscopic limited T-3 sympathicotomy for primary hyperhidrosis: prevention for compensatory hyperhidrosis: prevention for compensatory hiperhidrosis. J Neurosurg. 2003;99:39-43.

28. Singh B, Moodley J, Allopi L, Cassimjee HM. Horner syndrome after sympathectomy in the thoracoscopic era. Surg Laparosc Endosc Percutan Tech. 2006;16:222-5.

29. Lai YT, Yang LH, Chio CC, Chen HH. Complications in patients with palmar hyperhidrosis treated with transthoracic endoscopic sympathectomy. Neurosurgery. 1997;41:110-3; discussion 113-5.

30. Drott C, Gothberg G, Claes G. Endoscopic transthoracic sympathectomy: an efficient and safe method for the treatment of hyperhidrosis. J Am Acad Dermatol. 1995;33:78-81.

31. Riet M, Smet AA, Kuiken H, Kazemier G, Bonjer HJ. Prevention of compensatory hyperhidrosis after thoracoscopic sympathectomy for hyperhidrosis. Surg Endosc. 2001;15:1159-62.

32. Munia MA, Wolosker N, Kaufmann P, de Campos JR, PuechLeão P. Sustained benefit lasting one year from T4 instead of T3-T4 sympathectomy for isolated axillary hyperhidrosis. Clinics. 2008;63:771-4.

33. Wolosker N, Yazbek G, Ishy A, de Campos JR, Kauffman P, Puech-Leao $\mathrm{P}$. Is sympathectomy at $\mathrm{T} 4$ level better than at $\mathrm{T} 3$ level for treating palmar hyperhidrosis? J Laparoendosc Adv Surg Tech A. 2008;18:102-6.

34. Lau WT, Lee JD, Dang CR, Lee L. Improvement in quality of life after bilateral transthoracic endoscopic sympathectomy for palmar hyperhydrosis. Hawaii Med J. 2001;60:126-37.

35. Boley TM, Belangee KN, Markwell S, Hazelrigg SR. The effect of thoracoscopic sympathectomy on quality of life and symptom management of hyperhidrosis. J Am Coll Surg. 2007;204:435-8. 